\title{
Optical coherence tomography imaging after endovascular thrombectomy: a novel method for evaluating vascular injury in a swine model
}

\author{
Christopher R. Pasarikovski, MD, ${ }^{1}$ Julia Keith, MD, ${ }^{2}$ Leodante da Costa, MD, MSc, ${ }^{3}$ \\ Joel Ramjist, BSc, ${ }^{3}$ Yuta Dobashi, MSc, ${ }^{3}$ Sandra E. Black, OC, MD, FRCP(C), ${ }^{4,5}$ and \\ Victor X. D. Yang, MD, PhD1,3,4
}

'Division of Neurosurgery, Department of Surgery, University of Toronto; ${ }^{2}$ Department of Anatomic Pathology, Sunnybrook Health Sciences Centre, University of Toronto; ${ }^{3}$ Division of Neurosurgery, Sunnybrook Hospital, University of Toronto; ${ }^{4}$ Hurvitz Brain Sciences Research Program, Sunnybrook Research Institute, Sunnybrook Health Sciences Centre, University of Toronto; and ${ }^{5}$ Division of Neurology, Sunnybrook Health Sciences Centre, University of Toronto, Ontario, Canada

OBJECTIVE Although studies have shown that some degree of iatrogenic endothelial injury occurs during endovascular thrombectomy (EVT), the clinical significance of such injury is uncertain. Furthermore, it is likely that iatrogenic effects such as endothelial denudation, intimal dissection, and tunica media edema will have varying clinical implications. The purpose of this study was to assess the feasibility of endovascular optical coherence tomography (OCT) in quantifying vessel injury in real time after EVT, correlate vessel injury with histological findings, and perform imaging at varying time intervals after EVT to assess the impact of prolonged direct exposure of the vessel to the thrombus.

METHODS Yorkshire swine weighing 35-40 kg were selected for use as the animal model, with a total of 9 vessels from 3 swine examined. Thrombectomy was performed using a second-generation stent retriever 1, 3, and 6 hours after thrombus deposition. The presence and degree of denudation of the endothelium, detachment and separation of the layers of the tunic media, hemorrhage within the media, dissection of the vessels, and thrombus within the lumina were assessed using OCT images acquired immediately after EVT. Bland-Altman analysis indicated that these OCT findings were correlated with postmortem histological findings.

RESULTS OCT image acquisition was technically successful in all cases. Endothelial denudation was present in $65 \% \pm$ $16 \%, 87 \% \pm 8 \%$, and $93 \% \pm 7 \%$ of the vessel surface 1,3 , and 6 hours, respectively, after thrombus deposition and subsequent EVT. Residual intraluminal thrombus was present in vessels at all time intervals despite complete angiographic revascularization. Bland-Altman plots showed good agreement between OCT and histological analysis with respect to the degree of endothelial denudation and elevation, separation of the tunica media, and hemorrhage within the media. OCT appears to be more specific than histological analysis in detecting endothelial elevation.

CONCLUSIONS OCT is a feasible method that can be used to assess vascular injury after EVT with histological accuracy. Varying degrees of vessel injury occur after EVT, and residual luminal thrombus can be present despite complete angiographic revascularization.

https://thejns.org/doi/abs/10.3171/2019.12.JNS192881

KEYWORDS optical coherence tomography; endovascular thrombectomy; endothelium; thrombus; vascular disorders

$\mathrm{T}$ HE cerebrovascular endothelium is known to play a pivotal role in the regulation of inflammatory pathways, permeability of the blood-brain barrier, and thrombosis. ${ }^{11}$ During endovascular thrombectomy (EVT) with second-generation stent retrievers, considerable outward radial force can be delivered to the vessel wall via the stent struts or thrombus..$^{13}$ Studies evaluating iatrogenic endothelial injury during EVT have been performed by means of retrieved human thrombus, postmortem histopathologic analysis, magnetic resonance vessel-wall (MRVW) imaging, and animal histopathologic studies. ${ }^{4,14,18}$

ABBREVIATIONS AOL = arterial occlusive lesion; EVT = endovascular thrombectomy; ITA = internal thoracic artery; MRVW = magnetic resonance vessel wall; OCT = optical coherence tomography; $\mathrm{SCA}=$ superficial cervical artery; $\mathrm{TICl}=$ thrombolysis in cerebral infarction.

SUBMITTED October 22, 2019. ACCEPTED December 9, 2019.

INCLUDE WHEN CITING Published online February 14, 2020; DOI: 10.3171/2019.12.JNS192881. 
Furthermore, the mechanical thrombectomy device may not be the only source of damage to the endothelium during ETV. Prolonged vessel wall exposure to a luminal thrombus can also cause endothelial damage. ${ }^{22}$ Reil et al. showed that ligated arteries exposed to thrombus had increased endothelial damage compared with arteries with interrupted flow in the absence of thrombus. ${ }^{16}$ Various components of the thrombus can affect endothelial function and morphology?

Endovascular optical coherence tomography (OCT) imaging is the highest-resolution intravascular imaging modality currently available. This technology has traditionally been used in interventional cardiology, and more recently it has been applied in neurointerventional surgery. ${ }^{3}$ OCT utilizes near-infrared light with a wavelength of approximately $1.3 \mu \mathrm{m}$, and excellent intraluminal spatial resolutions of 10-20 $\mu \mathrm{m}$ are achievable with this method. ${ }^{19}$

Although studies have shown that some degree of iatrogenic endothelial injury likely occurs during EVT, whether this injury is clinically significant remains unknown. Before attempting to correlate vessel injury with clinical outcome, the source and degree of vessel wall injury should be adequately quantified, as it is likely that endothelial denudation, intimal dissection, and edema of the tunica media will have varying clinical implications. Currently available techniques have limitations, such as MRVW imaging, which has insufficient spatial resolution to allow direct visualization of endothelial injury, and histopathologic examinations, which are performed ex vivo, prone to processing artifacts, and cannot provide real-time patterns of injury. ${ }^{20}$ The purpose of this study was to assess the feasibility of endovascular OCT in quantifying vessel injury in real time after EVT, correlate the OCT findings of vessel injury with histological findings, and perform imaging after EVT at varying time intervals to assess the impact of prolonged direct vessel exposure to thrombus.

\section{Methods}

All experiments were conducted in accordance with policies established by our institutional research ethics board committee. Nine vessels in 3 Yorkshire swine (ordered from Caughell Farms, Ontario, Canada) weighing $35-40 \mathrm{~kg}$ were selected for the animal model, as these animals have well-developed superficial cervical arteries (SCA) similar in caliber to human middle cerebral arteries. ${ }^{25}$ All procedures were carried out under general anesthetic with continuous hemodynamic monitoring. The animals were fed standard diets at our facility for 2 weeks before the procedure.

\section{Technical Success Definition}

Technical parameters included performance of the following procedures: 1) navigating the OCT catheter to the appropriate location within the vessel, 2) clearing luminal blood with minimal scattering artifact from red blood cells, 3) capturing circumferential OCT images of the entire arterial lumen along the entire region of interest, 4) identifying normal anatomical structures (tunica intima, tunica media, adventitia, and internal/external elastic lam- ina) where present, and 5) identifying interatrial and vessel wall lesions when present. For the image acquisition to be defined as technically successful, all above criteria must have been met for each attempt.

\section{Thrombus Preparation}

Autologous venous whole blood was drawn into circular tubes without sodium citrate 48 hours before the procedure. ${ }^{12}$ The autologous blood was left to incubate at $4{ }^{\circ} \mathrm{C}$ for 24 hours. The thrombus (a solid red clot) was separated from the plasma. Before the procedure the thrombus was sectioned into $5 \times 20-\mathrm{mm}$ segments and stored at $37^{\circ} \mathrm{C}$.

\section{Vessel Occlusion and Thrombectomy}

An 8-Fr sheath was inserted into the right common femoral artery and connected to continuous saline irrigation. No heparin or other antithrombotic/thrombolytic agents were administered throughout the procedure. There is inconsistency among the major EVT trials with respect to procedural heparin administration. Kreitzer et al. reported that no two major EVT trials had the same heparin EVT protocol, and therefore we did not administer antithrombotics. ${ }^{8}$ First, a thrombus was deposited into each of 3 arteries sequentially as follows: 1) right SCA, 2) right internal thoracic artery (ITA), and 3) left SCA. A FlowGate (Stryker) double-lumen balloon guide catheter was positioned in the subclavian artery. A distal access catheter was navigated through the double-lumen balloon guide into the vessel of interest and the thrombus was injected slowly through the catheter under proximal balloon occlusion. If incomplete vessel occlusion was observed, thrombus injection was repeated until complete occlusion was observed.

EVT was then performed at 1,3, and 6 hours after vessel occlusion. A FlowGate guide catheter was again positioned in the subclavian artery. A Trevo-18 microcatheter (Stryker) was advanced over a microwire $3 \mathrm{~mm}$ beyond the thrombus. A Trevo $4 \times 20-\mathrm{mm}$ stent retriever (Stryker) was deployed for 5 minutes before retrieval. The thrombectomy devices were slowly withdrawn under proximal balloon occlusion and continued aspiration through a 60-ml syringe. The goal was complete revascularization, defined as thrombolysis in cerebral infarction (TICI) reperfusion grade 3 and arterial occlusive lesion (AOL) recanalization score of 3. If incomplete angiographic revascularization was observed, the procedure was repeated again for up to a maximum of 3 attempts.

\section{OCT Image Acquisition and Analysis}

After thrombectomy, OCT images were immediately obtained. The Dragonfly OCT catheter (Abbott Vascular) was used. The following 3 steps were sequentially followed for image acquisition: 1) loading an automated injection pump with $150 \mathrm{ml}$ saline to clear the blood within the lumen during image acquisition, 2) mounting the catheter on a rapid exchange microguidewire and positioning the device such that the optical lens radiopaque marker was distal to the segment with the previous thrombus, and 3) administering a saline infusion of $4 \mathrm{ml} / \mathrm{sec}$ for 6 seconds via the automated pump. The OCT catheter auto- 
matically detects clearing of luminal blood and performs a motorized automated pullback of $54 \mathrm{~mm}$ total for image acquisition. OCT imaging frequency is 100 frames/sec, with a total of 540 cross-sectional images generated per acquisition attempt.

All cross-sectional OCT images were analyzed. The percentage of surface area was used to quantify endothelial injury, and the inner tunica media circumference percentage was used to determine media layer edema and separation. The following characteristics of vascular injury were quantified: 1) endothelial denudation, 2) endothelial detachment and elevation from the underlying media, 3) separation of layers of the tunica media, 4) hemorrhage within the media, 5) vessel dissection, and 6) luminal thrombus. In order to quantify the degree of residual thrombus present, the thrombus scoring system developed by Prati et al. was utilized. ${ }^{15}$ This scoring system is based first on the semiquantitative assessment of the number of quadrants with residual thrombus present in each crosssectional OCT image. A value of zero is assigned if no residual thrombus is present. A value of $1,2,3$, or 4 is assigned to each cross-sectional OCT image depending on the number of quadrants with thrombus. Subsequently, all scores from each cross-section are added for a final thrombus burden total score.

\section{Histological Analysis}

After OCT imaging was completed and the animal was humanely killed, an approximately 4-cm segment of each affected artery was harvested. The site of the thrombus was landmarked using angiography images and marked on the external surface of the artery using tissue ink. The resected arterial segments were fixed in $10 \%$ neutral buffered formalin. After formalin fixation, the arteries were cross-sectioned into 5-mm segments and embedded in paraffin. Three different levels through each of these tissue blocks were created, and 5- $\mu \mathrm{m}$-thick tissue sections were mounted on glass slides and stained with $\mathrm{H} \& \mathrm{E}$. The H \& E-stained slides from each level of the sampled arteries were examined at $4 \times, 10 \times$, and $20 \times$ magnification by an experienced neuropathologist (J.K.), and the same 6 characteristics of vascular injury were assessed (endothelial denudation, endothelial detachment, separation of layers of the media, hemorrhage within the media, dissection, and luminal thrombus). The only difference arose in the assessment of residual luminal thrombus. On histological analysis, thrombus quantification was defined as present or absent, whereas with OCT the method by Prati et al. was utilized as described above. ${ }^{15}$ Slides were scanned by using an Aperio AT Turbo slide scanner (Leica Biosystems), and the dimensions of the luminal thrombi, mural thrombi, and dissection were measured in micrometers.

\section{Statistical Analysis}

Bland-Altman plots were generated using the mean difference between histological findings and OCT scores and a $1.96 \mathrm{SD}$ (corresponding to 2 SDs in either direction) with respect to the various vessel wall injury characteristics. All statistics were calculated using SPSS version 25 (IBM).
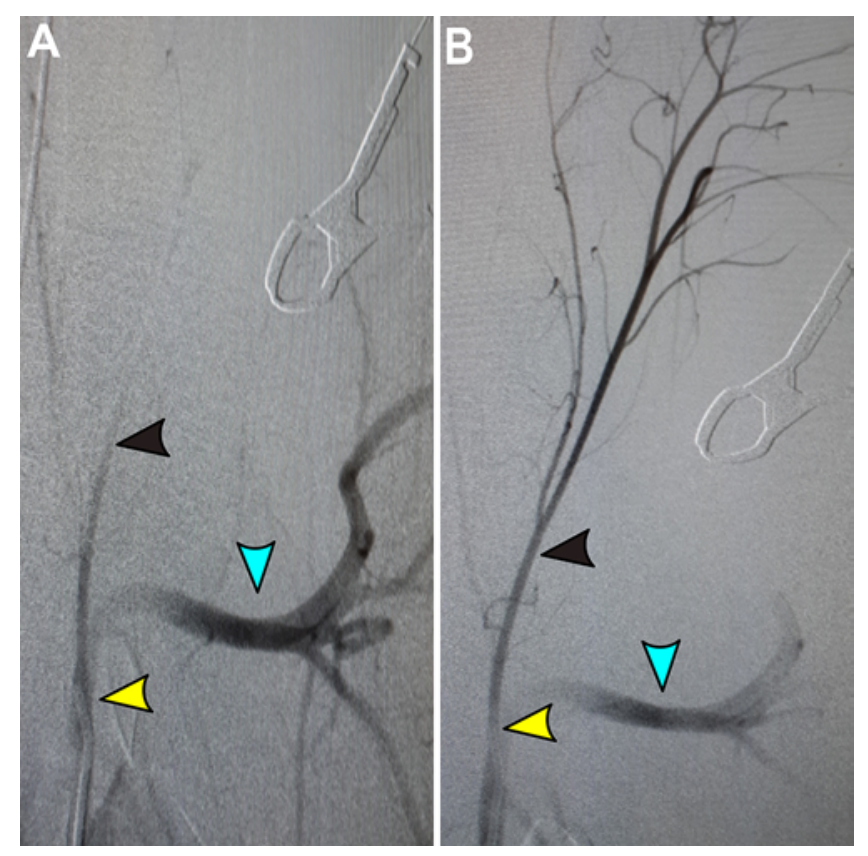

FIG. 1. Clot deposition and thrombectomy. A catheter is shown in the proximal left subclavian artery (yellow arrow) with filling of the middle left subclavian artery (blue arrow). A: Occlusion of the proximal left superficial cervical artery (black arrow) using an erythrocyte-rich thrombus. B: The left SCA is patent (TICI/AOL 3 revascularization) after mechanical thrombectomy (black arrow). Figure is available in color online only.

\section{Results}

Successful revascularization (TICI/AOL grade 3) was achieved in all 9 vessels (Fig. 1). OCT image acquisition was technically successful in all (100\%) cases. Regions beyond the thrombectomy site showed normal swine vessel anatomy, with a thin layer of endothelium and thick tunica media, which constitutes most of the arterial wall, and no obvious external elastic lamina (Fig. 2A). On OCT analysis, endothelial denudation (Fig. 2C) was present in $65 \%$ $\pm 16 \%, 87 \% \pm 8 \%$, and $93 \% \pm 7 \%$ of the vessel surface 1 , 3 , and 6 hours, respectively, after thrombus deposition and subsequent EVT (Table 1). Separation of the layers within the media was present in $5 \% \pm 15 \%, 49 \% \pm 11 \%$, and $66 \%$ $\pm 18 \%$ of the vessels 1,3 , and 6 hours, respectively, after thrombus deposition and subsequent EVT. Hemorrhage was observed within the media only for vessels exposed to thrombus for 6 hours. Dissection was present in only 1 vessel exposed to thrombus for 6 hours (Fig. 2D). Residual intraluminal thrombus was present in vessels at all time intervals despite complete angiographic revascularization (TICI/AOL grade 3). The residual thrombus burden scores were $6.2 \pm 1,4.3 \pm 2$, and $9 \pm 5$ in vessels 1,3 , and 6 hours, respectively, after thrombus deposition and subsequent EVT (Fig. 3A-D). The average numbers of EVT passes to achieve TICI/AOL grade 3 reperfusion were 1.3, 1 , and 2 after 1,3 , and 6 hours, respectively.

Bland-Altman analysis demonstrated good agreement between histological results (Fig. 4) and OCT images across all vessel injury characteristics (Fig. 5). For endothelial denudation, a mean difference with 1.96 and 

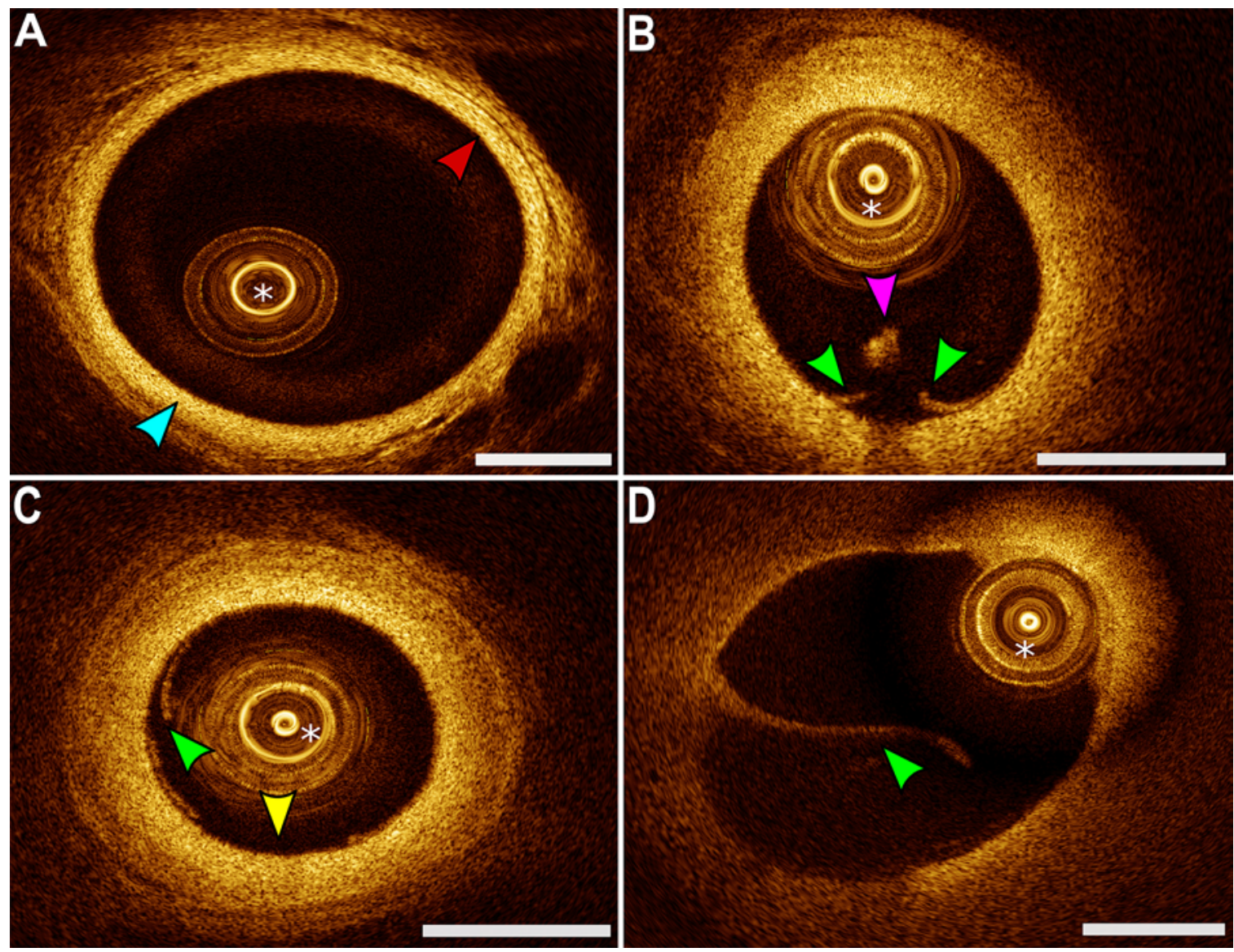

FIG. 2. OCT imaging of normal and damaged swine endothelium (asterisk indicates OCT catheter). A: Normal swine vessel anatomy with thick tunica media occupying most of the vessel wall (blue arrow), with a thin layer of endothelial cells (red arrow). B: Damaged endothelial layer (green arrows) elevated from the tunica media with an intraluminal thrombus (pink arrow). C: Elevated endothelium (green arrow) and denuded endothelium (yellow arrow). D: Floating tunica intima and tunica media within the vessel lumen (green arrow). Bar $=1 \mathrm{~mm}$. Figure is available in color online only.

$-1.96 \mathrm{SD}$ (mean [SD] of $0.11[0.29,-0.3]$ ) was observed, with 5\% (4/73) of points outside the 95\% CI (Fig. 5A). For endothelial elevation a mean difference of $0.001(0.04$, $-0.04)$ was observed, with $4 \%$ (3/73) of points outside the 95\% CI (Fig. 5B). For separation of the media, a mean difference of $-0.04(0.27-0.36)$ was observed, with $8 \%$ (6/73) of points outside the 95\% CI (Fig. 5C). For hemorrhage of the media a mean difference of $0.01(0.15,-0.16)$ was observed, with 7\% (4/59) of points outside the $95 \%$ CI (Fig. 5D).

\section{Discussion}

We describe what is to our knowledge the first use of OCT in a technique for in vivo imaging of the vessel wall and luminal environment after EVT. Our findings include the following observations: 1) OCT imaging is technically feasible and can adequately quantify vessel wall injury, 2) varying degrees of vessel injury occur after EVT, 3) residual luminal thrombus can be present despite complete angiographic revascularization (TICI/AOL grade 3), and 4) prolonged direct vessel exposure to thrombus before EVT may increase vessel injury.
Endovascular thrombectomy has become the standard of care after several large randomized trials showed efficacy for patients with large vessel occlusion of the anterior circulation. ${ }^{5}$ Yuki et al. and Gori et al. in 2013 first reported a novel animal model to evaluate iatrogenic arterial injury during mechanical thrombectomy by exposing the target arteries, implanting the thrombi, performing mechanical thrombectomy, and conducting histological assessment after harvesting the vessels.,25 These techniques have limitations, as the vessels need to be exposed and manipulated, with a change in the surrounding soft tissue environment, and more importantly, histological analysis may be prone to processing artifacts. To circumvent these limitations, Teng et al. in 2015 developed an in vitro livecell platform to assess thrombectomy devices and allow direct visualization of the cell-device interaction..$^{20}$ Along with being in vitro, these platforms examine only endothelial injury and not the entire vessel wall.

Although histological analysis remains the gold standard in the characterization of vascular injury, OCT, with a spatial resolution of $10 \mu \mathrm{m}$, clearly defines the different layers of the arterial wall and enables assessment of the luminal environment without tissue preparation. We found 
Pasarikovski et al.

TABLE 1. Histological and OCT quantification of vessel wall injury

\begin{tabular}{cccccccc}
\hline $\begin{array}{c}\text { Quantification } \\
\text { Method }\end{array}$ & $\begin{array}{c}\text { Hrs After Thrombus } \\
\text { Deposition \& EVT }\end{array}$ & $\begin{array}{c}\text { Endothelial } \\
\text { Denudation }\end{array}$ & $\begin{array}{c}\text { Endothelial } \\
\text { Elevation }\end{array}$ & $\begin{array}{c}\text { Tunica Media } \\
\text { Separation }\end{array}$ & $\begin{array}{c}\text { Tunica Media } \\
\text { Hemorrhage }\end{array}$ & $\begin{array}{c}\text { Residual } \\
\text { Dissection }\end{array}$ & $\begin{array}{c}\text { Thrombus } \\
\text { Histology }\end{array}$ \\
\hline Lt SCA & 1 & $70 \pm 10 \%$ & $3 \pm 4 \%$ & $1 \pm 2 \%$ & 0 & No & Yes \\
\hline Rt ITA & 3 & $82 \pm 15 \%$ & 0 & $43 \pm 15 \%$ & $1 \pm 3 \%$ & No & Yes \\
\hline Rt SCA & 6 & $85 \pm 28 \%$ & $8 \pm 27 \%$ & $72 \pm 27 \%$ & $12 \pm 40 \%$ & Yes & Yes \\
\hline OCT & & & & & & & \\
\hline Lt SCA & 1 & $65 \pm 16 \%$ & $7 \pm 5 \%$ & $5 \pm 15 \%$ & 0 & No & $6.2 \pm 1$ \\
\hline Rt ITA & 3 & $87 \pm 8 \%$ & 0 & $49 \pm 11 \%$ & 0 & No & $4.3 \pm 2$ \\
\hline Rt SCA & 6 & $93 \pm 7 \%$ & $20 \pm 15 \%$ & $66 \pm 18 \%$ & $21 \pm 16 \%$ & Yes & $9 \pm 5$ \\
\hline
\end{tabular}

Endothelial injury, residual thrombus, and edema within the tunica media were present in all vessels.

OCT image acquisition to be feasible in all cases with the use of our standardized protocol. Endothelial denudation was certainly quantifiable, along with the presence of luminal thrombus. Supporting this, Bland-Altman analysis confirmed acceptable agreement between histology and OCT for the varying degrees of vascular injury. It also appears that OCT can quantify endothelial elevation with increased specificity. We hypothesize that the use of histological specimens, which are analyzed ex vivo and prone to subtle processing artifacts, may lead to underestimation of the degree of endothelial elevation. Furthermore, the novel technique we report here could address the deficiencies of prior models utilized to evaluate thrombectomy devices. Currently, most devices are tested in silicon and
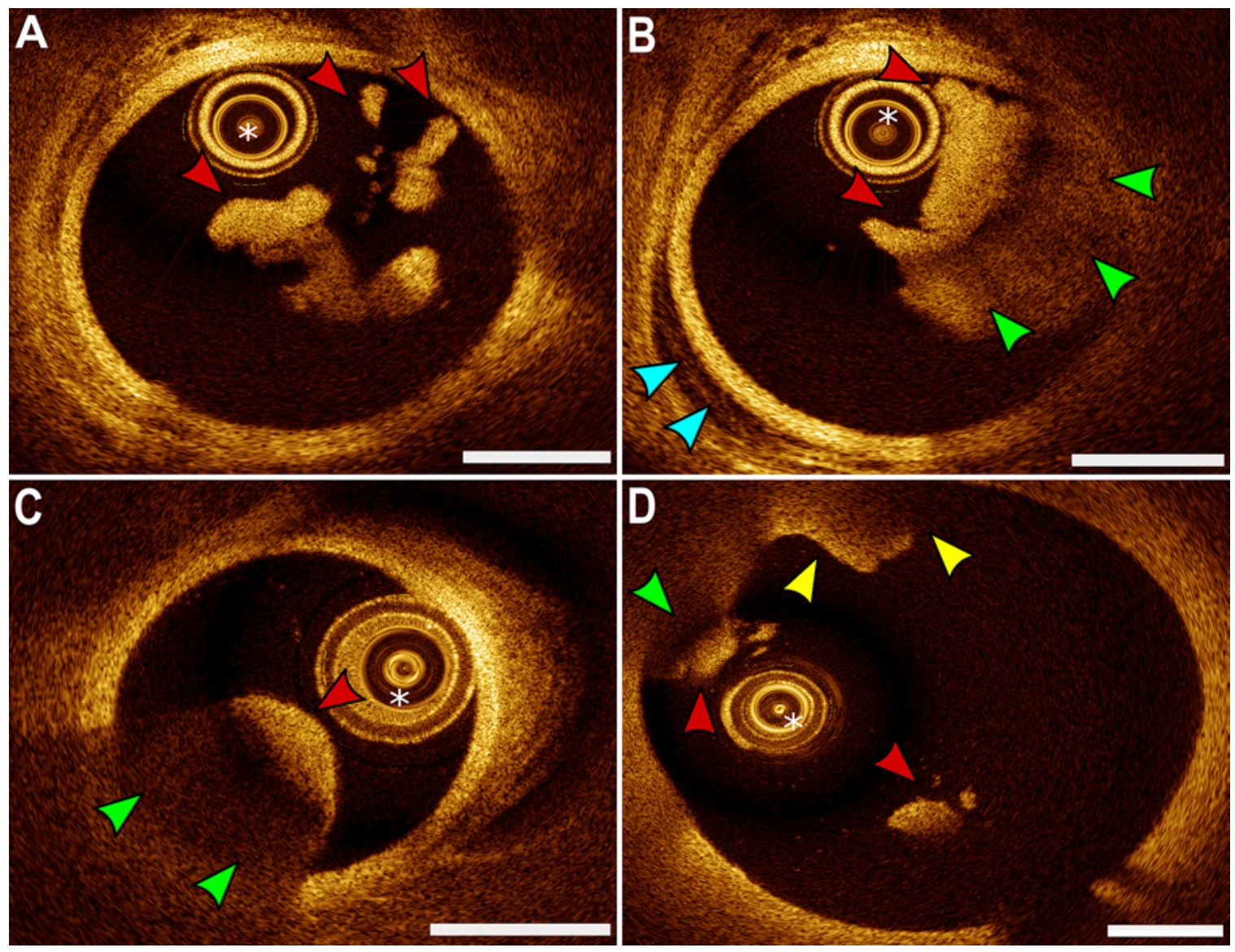

FIG. 3. OCT imaging revealing residual thrombus (asterisk indicates OCT catheter). A-D: Intraluminal residual thrombus (red arrows) with shadowing beyond the red thrombus (green arrows) despite TICI/AOL grade 3 revascularization. B: Fluid and expansion observed within the tunica media (blue arrows). D: Thrombus within the vessel wall (yellow arrows) extending into the lumen. Bar = $1 \mathrm{~mm}$. Figure is available in color online only. 

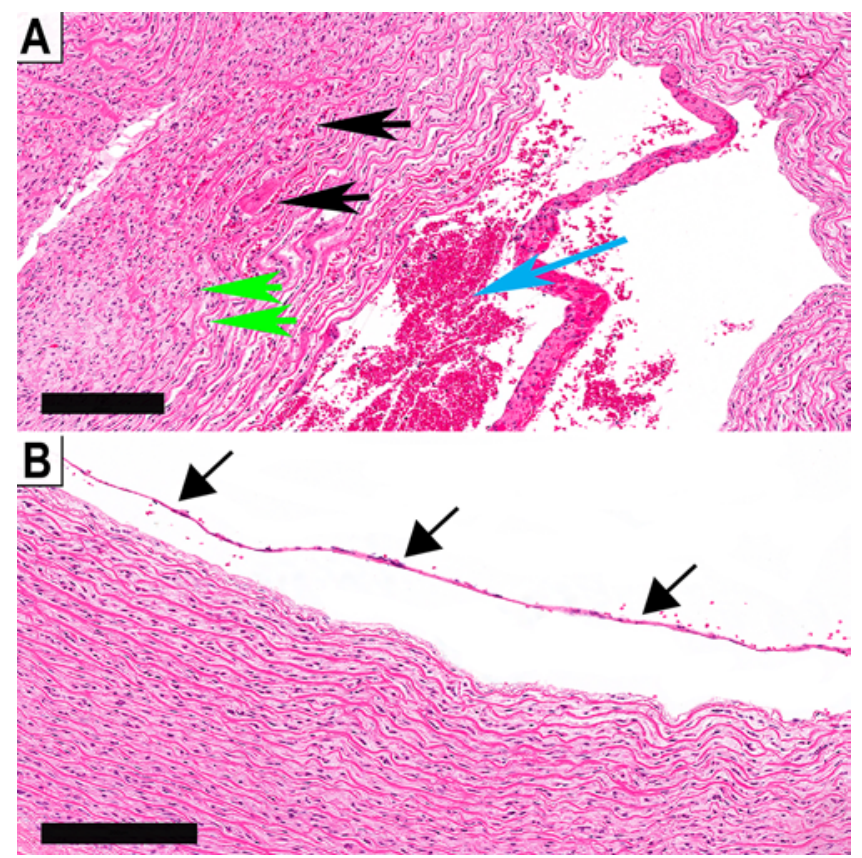

FIG. 4. Histological analysis showing vessel injury. A: Vessel dissection (blue arrow), hemorrhage within the tunica media (black arrow), and neutrophil infiltration (green arrows). B: Endothelial elevation (black arrows) from the tunica media. Bar $=200 \mu \mathrm{m}$. Figure is available in color online only. glass phantom models to allow for direct visualization, and these models lack a biologically representative environment. OCT can specifically address this shortcoming and allow for observation of the interaction between the device and vessel wall in vivo.

The clinical significance of vessel wall injury during EVT remains unknown. The largest study investigating the relationship between vessel wall injury and clinical outcome was performed by Renú et al. in 2017 by means of MRVW imaging on a $1.5 \mathrm{~T}$ scanner. ${ }^{17}$ These authors found that 34 patients $(57 \%)$ had gadolinium vessel wall enhancement after EVT, which was associated with poor clinical outcome. The insufficient spatial resolution of MRVW imaging limits this modality to use for assessing contrast enhancement or mural hemorrhage and cannot enable adequate visualization of varying degrees of injury. Direct evidence of vessel injury in humans was reported by Yin et al., who performed autopsies in 5 patients after EVT and found subintimal dissection in 1 patient that likely resulted from the procedure. ${ }^{24}$ We hypothesized that varying degrees of injury (endothelial denudation, intimal dissection, and edema of the media) will have varying clinical implications, and that OCT will enable us to quantify these degrees of injury in real time.

An unexpected finding was the presence of residual luminal thrombus despite complete angiographic revascularization. Chueh et al. demonstrated that distal embolic showers with large $(215-285 \mu \mathrm{m})$ and small fragments
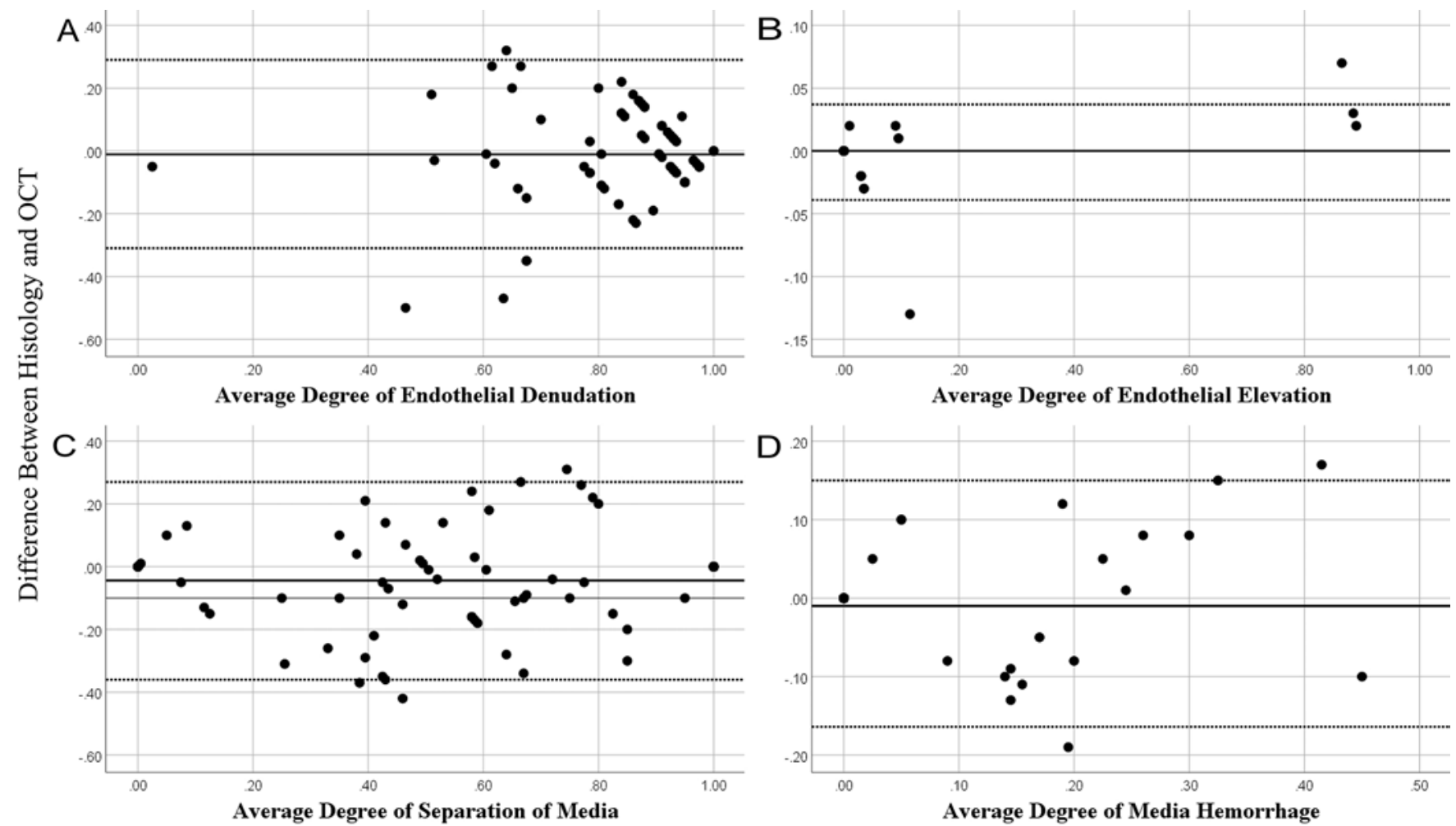

FIG. 5. Bland-Altman plots comparing histology and OCT with respect to different characteristics of vessel wall injury. A: Endothelial denudation with a mean difference SD $(1.96,-1.96)$ of $0.11(0.29,-0.31)$. B: Endothelial elevation with a mean difference of $0.001(0.04,-0.04)$. C: Separation of the tunica media with a mean difference of $0.04(0.27,-0.36)$. D: Hemorrhage of the media with a mean difference of $0.01(0.15,-0.16)$. 
(23-37 $\mu \mathrm{m})$ occur during thrombectomy. ${ }^{1}$ Although distal emboli have certainly been reported, to our knowledge there are no reports in the literature of residual luminal thrombi at the site of the target lesion in the presence of complete recanalization and reperfusion (TICI/AOL grade 3). Gory et al. reported significant mural thrombus at the thrombectomy site; however, these lesions were in the intimal layer and were causing no luminal narrowing. ${ }^{2}$ These authors hypothesized that the observed thrombus was a combination of residual clot not captured by the stent retriever, along with new aggregations of platelets at the thrombectomy site due to exposed collagen from vessel wall injury. The clinical implications of residual thrombus after EVT remain unknown. Our recent case series including 3 consecutive patients with basilar occlusion revealed significant residual thrombus adjacent to patent basilar perforators in 2 patients after EVT. ${ }^{10}$ The thrombus was not visible on cerebral angiography, CT angiography, or MRVW imaging and could certainly cause ongoing strokes.

To our knowledge, there have been no reported studies evaluating endothelial injury at increasing time points to investigate the possible contribution of prolonged vessel wall exposure to thrombus in endothelial injury. Power et al. showed that thromboembolism alone can cause MRVW image abnormalities in the absence of EVT in 30\% ( $=$ 3) patients treated with medical therapy alone. ${ }^{14}$ These authors found that patients undergoing EVT had significantly increased arterial wall abnormality compared to those treated with medical therapy alone, suggesting that iatrogenic injury occurs from the thrombectomy device. In the present study, we observed increased endothelial injury and intimal edema in vessels undergoing EVT 6 hours after thrombus deposition. Components of the thrombus can affect endothelial function and morphology, and we also hypothesize that the clot may be more adherent to the vessel wall after increased time, causing more mechanical injury during retrieval.?

\section{Study Limitations}

There are several limitations to our model. First, swine extracranial arteries differ anatomically from human cerebral vessels. Swine blood vessels generally have a thicker tunica media and a thinner intimal layer than human vessels. ${ }^{25}$ Furthermore, swine vessels are more elastic than human vessels. ${ }^{21}$ This vessel elasticity in the swine model can result in underestimation of the degree of vessel wall injury due to stress and strain forces, as more elastic vessels can better tolerate these forces, leading to decreased dissection and tears. However, OCT appears to be feasible in quantifying injury in the animal model and should also be feasible in quantifying injury in humans. Second, we used erythrocyte-rich thrombus in our model, which differs slightly from the cellular composition of thromboemboli in the clinical setting. Third, the swine vessels utilized in our model were nontortuous and the vascular access was straightforward. The human intracranial circulation has significant vessel tortuosity, particularly at the carotid siphon, and navigation of the OCT catheter may not be feasible for all patients. The distal tip of the OCT catheter is stiff due to the reflecting glass lens. In the authors' own institutional experience, OCT image acquisition of the posterior circulation is straightforward. However, overly tortuous carotid siphons are not easily traversed. Other authors have reported techniques for reliable navigation of tortuous carotid siphons. ${ }^{6,9}$ Finally, the OCT catheter itself could also be a source of vascular injury. Large safety and feasibility studies reported in the interventional cardiology literature suggest that intravascular OCT is safe; however, the intracranial vasculature is unique, and specific investigations with respect to the intracranial circulation will need to be undertaken to ensure the safety of intravascular OCT in the setting of intracranial vasculature. ${ }^{23}$

\section{Conclusions}

Varying degrees of vessel injury occur after EVT and residual luminal thrombus can be present despite complete angiographic revascularization. OCT imaging after EVT was demonstrated to be feasible in this preclinical study and can adequately quantify vessel wall injury in real time with histological accuracy.

\section{References}

1. Chueh JY, Wakhloo AK, Gounis MJ: Effectiveness of mechanical endovascular thrombectomy in a model system of cerebrovascular occlusion. AJNR Am J Neuroradiol 33:1998-2003, 2012

2. Gory B, Bresson D, Kessler I, Perrin ML, Guillaudeau A, Durand K, et al: Histopathologic evaluation of arterial wall response to 5 neurovascular mechanical thrombectomy devices in a swine model. AJNR Am J Neuroradiol 34:21922198,2013

3. Gounis MJ, Ughi GJ, Marosfoi M, Lopes DK, Fiorella D, Bezerra HG, et al: Intravascular optical coherence tomography for neurointerventional surgery. Stroke 50:218-223, 2019

4. Gounis MJ, Wakhloo AK, Chueh JY: Preclinical investigations for thrombectomy devices - does it translate to humans? Stroke 44 (6 Suppl 1):S7-S10, 2013

5. Goyal M, Menon BK, van Zwam WH, Dippel DW, Mitchell PJ, Demchuk AM, et al: Endovascular thrombectomy after large-vessel ischaemic stroke: a meta-analysis of individual patient data from five randomised trials. Lancet 387:17231731, 2016

6. Guerrero BP, Pacheco CD, Saied A, Joshi K, Rodríguez C, Martínez-Galdámez M, et al: First human evaluation of endothelial healing after a Pipeline flex embolization device with shield technology implanted in posterior circulation using optical coherence tomography. Neurointervention 13:129-132, 2018

7. Jørgensen L, Grøthe AG, Larsen T, Kinlough-Rathbone RL, Mustard JF: Injury to cultured endothelial cells by thrombinstimulated platelets. Lab Invest 54:408-415, 1986

8. Kreitzer N, Khatri P, Adeoye O, Abruzzo T, Grossman A, Ringer A, et al: Abstract TP2: heparin use across major endovascular trials. Stroke 47:ATP2, 2016

9. Martínez-Galdámez M, Escartín J, Pabón B, Diaz C, MartínReyes R, Hermosín A, et al: Optical coherence tomography: translation from 3D-printed vascular models of the anterior cerebral circulation to the first human images of implanted surface modified flow diverters. Interv Neuroradiol 25:150 156, 2019

10. Pasarikovski CR, Ramjist J, da Costa L, Black SE, Yang V: Optical coherence tomography imaging after endovascular thrombectomy for basilar artery occlusion: report of 3 cases. J Neurosurg [epub ahead of print August 23, 2019. DOI: 10.3171/2019.5.JNS191252] 
11. Pearson JD: Endothelial cell function and thrombosis. Best Pract Res Clin Haematol 12:329-341, 1999

12. Peschillo S, Diana F, Berge J, Missori P: A comparison of acute vascular damage caused by ADAPT versus a stent retriever device after thrombectomy in acute ischemic stroke: a histological and ultrastructural study in an animal model. J Neurointerv Surg 9:743-749, 2017

13. Pierot L, Soize S, Benaissa A, Wakhloo AK: Techniques for endovascular treatment of acute ischemic stroke: from intraarterial fibrinolytics to stent-retrievers. Stroke 46:909-914, 2015

14. Power S, Matouk C, Casaubon LK, Silver FL, Krings T, Mikulis DJ, et al: Vessel wall magnetic resonance imaging in acute ischemic stroke: effects of embolism and mechanical thrombectomy on the arterial wall. Stroke 45:2330-2334, 2014

15. Prati F, Capodanno D, Pawlowski T, Ramazzotti V, Albertucci M, La Manna A, et al: Local delivery versus intracoronary infusion of abciximab in patients with acute coronary syndromes. JACC Cardiovasc Interv 3:928-934, 2010

16. Reil TD, Moore WS, Kashyap VS, Nene SS, Gelabert HA, Quinones-Baldrich WJ: The effects of thrombus, thrombectomy and thrombolysis on endothelial function. Eur J Vasc Endovasc Surg 19:162-168, 2000

17. Renú A, Laredo C, Lopez-Rueda A, Llull L, Tudela R, SanRoman L, et al: Vessel wall enhancement and blood-cerebrospinal fluid barrier disruption after mechanical thrombectomy in acute ischemic stroke. Stroke 48:651-657, 2017

18. Singh P, Doostkam S, Reinhard M, Ivanovas V, Taschner CA: Immunohistochemical analysis of thrombi retrieved during treatment of acute ischemic stroke: does stent-retriever cause intimal damage? Stroke 44:1720-1722, 2013

19. Tearney GJ, Regar E, Akasaka T, Adriaenssens T, Barlis P, Bezerra HG, et al: Consensus standards for acquisition, measurement, and reporting of intravascular optical coherence tomography studies: a report from the International Working Group for Intravascular Optical Coherence Tomography Standardization and Validation. J Am Coll Cardiol 59:1058-1072, 2012 (Erratum in J Am Coll Cardiol 59:1662, 2012)

20. Teng D, Pannell JS, Rennert RC, Li J, Li YS, Wong VW, et al: Endothelial trauma from mechanical thrombectomy in acute stroke: in vitro live-cell platform with animal validation. Stroke 46:1099-1106, 2015
21. van Andel CJ, Pistecky PV, Borst C: Mechanical properties of porcine and human arteries: implications for coronary anastomotic connectors. Ann Thorac Surg 76:58-65, 2003

22. Wengrovitz M, Healy DA, Gifford RR, Atnip RG, Thiele BL: Thrombolytic therapy and balloon catheter thrombectomy in experimental femoral artery thrombosis: effect on arterial wall morphology. J Vasc Interv Radiol 6:205-210, 1995

23. Yamaguchi T, Terashima M, Akasaka T, Hayashi T, Mizuno $\mathrm{K}$, Muramatsu T, et al: Safety and feasibility of an intravascular optical coherence tomography image wire system in the clinical setting. Am J Cardiol 101:562-567, 2008

24. Yin NS, Benavides S, Starkman S, Liebeskind DS, Saver JA, Salamon N, et al: Autopsy findings after intracranial thrombectomy for acute ischemic stroke: a clinicopathologic study of 5 patients. Stroke 41:938-947, 2010

25. Yuki I, Kan I, Golshan A, Sohn J, Murayama Y, Vinters HV, et al: A swine model to analyze arterial structural changes induced by mechanical thrombectomy. AJNR Am J Neuroradiol 34:E87-E90, 2013

\section{Disclosures}

The authors report no conflict of interest concerning the materials or methods used in this study or the findings specified in this paper

\section{Author Contributions}

Conception and design: Yang, Pasarikovski, da Costa, Black. Acquisition of data: Yang, Pasarikovski, da Costa, Ramjist, Dobashi. Analysis and interpretation of data: all authors. Drafting the article: all authors. Critically revising the article: Yang, Keith, Ramjist, Dobashi, Black. Reviewed submitted version of manuscript: Pasarikovski, Keith, da Costa, Ramjist, Dobashi, Black. Approved the final version of the manuscript on behalf of all authors: Yang. Statistical analysis: Pasarikovski. Administrative/ technical/material support: Keith. Study supervision: Yang.

\section{Correspondence}

Victor X. D. Yang: Sunnybrook Health Sciences Centre, University of Toronto, ON, Canada. victor.yang@utoronto.ca. 